\title{
EL "POST" Y EL "TRANS" COMO ESTRUCTURAS CONSTITUTIVAS DE LA CULTURA: HACIA UNA PERSPECTIVA ERRÁTICA DE LA VIDA HUMANA ${ }^{1}$
}

\author{
Miguel Ángel Villamil Pineda ${ }^{2}$ \\ José Miguel Cabarcas Bolaños ${ }^{3}$ \\ Rubén Darío Vallejo Molina ${ }^{4}$ \\ Wilson Hernando Soto Urrea ${ }^{5}$ \\ Alberto René Ramírez Téllez ${ }^{6}$
}

Resumen: El presente artículo tiene como propósito analizar el significado de los prefijos "post" y "trans" en el marco del discurso académico sobre la cultura contemporánea. Al respecto, conviene preguntar: ¿Cuál es el referente cultural de estos prefijos? Nuestra hipótesis es que ambos prefijos hacen referencia a tres dinamismos culturales fundamentales, a saber: 1). El reconocimiento de los

1 Este artículo es resultado del proyecto de investigación "El juego como mediación pedagógica para el cultivo de la paz", código 19015020, aprobado por la Unidad de Investigación para el Fomento y Desarrollo de la Investigación de alto nivel de la Universidad Santo Tomás, Colombia. Una versión preliminar el texto, fue presentada como conferencia, el día 9 de septiembre de 2017, en el V Simposio Internacional Dialogos na Contemporaneidade, celebrado en la Universidad Univates - Brasil.

2 Doctor en Filosofía de la Pontificia Universidad Javeriana de Bogotá, Colombia; Magíster en Filosofía Latinoamericana y Licenciado en Filosofía y Letras de la Universidad Santo Tomás. Actualmente se desempeña como docente e investigador del Departamento de Humanidades y Formación Integral de la Universidad Santo Tomás. Dirección electrónica: miguel.villamil@ usantotomas.edu.co

3 Doctor de la Facultad de Ciencias Exactas de la Universidad Nacional de La Plata, Argentina. Físico de la Universidad Nacional de Colombia. E-mail: josecabarcasl@usantotomas.edu.co.

4 Doctor en Educación de la Universidad de la Salle de Costa Rica; Magíster en Educación de la Universidad Santo Tomás y Licenciado en Ciencias de la Educación en Lingüística y Literatura de la Universidad Distrital Francisco José de Caldas. Email: rubenvallejo@usantotomas.edu.co.

5 Doctor y Magíster en Educación de la Universidad Pedagógica Nacional e Ingeniero Mecánico de la Universidad Nacional de Colombia. E-mail: wilsonsoto@usantotomas.edu.co.

6 Doctor en Filosofía de la Universidad Nacional de Educación a Distancia de España; Magíster en Teología de la Universidad Javeriana; Magíster en Filosofía Latinoamericana y Licenciado en Filosofía de la Universidad Santo Tomás de Colombia. E-mail: frayalbertoramirez@usantotomas.edu.co 
límites de normatividades culturales establecidas; 2). La actitud crítica, disruptiva o transgresora frente a dichas normatividades; 3). El vislumbramiento o la constatación de nuevas significaciones, potencialidades o posibilidades culturales. En consecuencia, consideramos que los prefijos "post" y "trans" no son una "moda" de la narrativa actual, sino expresiones de la dinámica errática (errante) de la cultura (Jaramillo et al., 2018; Sáez, 2009, 2015; Villamil, 2017). Para argumentar la hipótesis planteada, proponemos el siguiente recorrido: inicialmente, presentaremos la vida humana como el dinamismo entre dos potencias heterogéneas y correlativas: la potencia céntrica y la potencia excéntrica. Luego, señalaremos los centrismos y los excentricismos culturales como patologías que ponen en crisis la cultura. Después, mostraremos el dinamismo errático de la vida humana como un criterio válido para la valoración de las culturas saludables, esto es, culturas que dinamicen tanto el poder normativo de la centricidad, como el poder creativo de la excentricidad. Finalmente, sostendremos que el cuidado y promoción del dinamismo errático de la cultura puede ser considerado como un criterio idóneo para la valoración ética de los movimientos "post" y "trans" contemporáneos.

Palabras clave: Post. Trans. Cultura. Errático. Centricidad. Excentricidad. Normatividad. Criterio.

\title{
THE "POST" AND THE “TRANS" AS CONSTITUTIVE STRUCTURES OF CULTURE: TOWARDS AN ERRATIC PERSPECTIVE OF HUMAN LIFE
}

\begin{abstract}
The purpose of this article is to analyze the meaning of the "post" and "trans" prefixes in the context of academic discourse on contemporary culture. In this regard, it is worth asking: What is the cultural reference of these prefixes? Our hypothesis is that both prefixes refer to three fundamental cultural dynamisms, namely: 1). The recognition of the limits of established cultural normativities; two). The critical, disruptive or transgressive attitude towards these normativities; 3 ). The glimpse or the verification of new meanings, potentials or cultural possibilities. Consequently, we consider that the prefixes "post" and "trans" are not a "fad" of the current narrative, but expressions of the erratic (wandering) dynamics of culture (Jaramillo et al., 2018; Sáez, 2009, 2015; Villamil, 2017). To argue the hypothesis, we propose the following route: initially, we will present human life as the dynamism between two heterogeneous and correlative powers: the central power and the eccentric power. Then, we will point out centrisms and cultural eccentricisms as pathologies that put culture in crisis. Then, we will show the erratic dynamism of human life as a valid criterion for the valuation of healthy cultures, that is, cultures that dynamize both the normative power of centricity and the creative power of eccentricity. Finally, we will maintain that the care and promotion of the erratic dynamism of culture can be considered as an ideal criterion for the ethical evaluation of contemporary "post" and "trans" movements.
\end{abstract}

Keywords: Post. Trans. Culture. Erratic. Centricity Eccentricity. Normativity. Criterion.

\section{O "POST" E O "TRANS" COMO ESTRUTURAS CONSTITUTIVAS DA CULTURA: RUMO A UMA PERSPECTIVA ERRÁTICA DA VIDA HUMANA}

Resumo: O presente artigo tem o como propósito analisar o significado dos prefixos "post" e "trans" no contexto do discurso acadêmico sobre a cultura contemporânea. A partir daqui, é conveniente perguntar: Qual é a referência cultural desses prefixos? Nossa hipótese é que esses 
prefixos referemse a três dinâmicas culturais fundamentais, a saber: 1). $O$ reconhecimento dos limites das normatividades culturais estabelecidas; 2) A atitude crítica, perturbadora ou transgressora em relação a esses regulamentos; 3) A previsão ou verificação de novos significados, potenciais ou possibilidades culturais. Por consequência, consideramos os prefixos "post" e "trans" no som uma "moda" narrativa atual, mas expressóes da dinâmica errática (errante) da cultura (Jaramillo et al., 2018; Sáez, 2009, 2015; Villamil, 2017). Para justificar a hipótese plantada, propomos o seguinte: inicialmete, apresentaremos a vida humana como o dinamismo entre dois poderes heterogêneos e correlativos: o poder cêntrico e o poder excêntrico. A seguir, apontaremos os centrismos e os excentricismos culturais como patologias que apontam para uma crise na cultura. Depois disso, mostraremos o dinamismo errático da vida humana como um critério válido para a valorizaçáo de culturas saudáveis, culturas que energizam o poder normativo da centricidade e o poder criativo da excentricidade. Por fim, observaremos que o cuidado e a promoçáo do dinamismo errático da cultura podem ser considerados como um critério apropriado para a avaliação ética dos movimentos "post" e "trans" contemporâneos.

Palavras-chave: Post. Trans. Cultura. Errático. Centricidade. Excentricidade. Normatividade. Critério.

\section{Introducción}

En la narrativa académica contemporánea hay dos prefijos que han ganado popularidad, a saber: "post" y "trans". El prefijo "post" ha sido utilizado como acompañante de una variedad de temas como los siguientes: postmodernidad, postmetafísica, postsecularismo, postcolonialismo, posthumanismo, postestructuralismo, postfeminismo, postconflicto, postporno, postverdad, entre otros. El significado del término "post" parece hacer referencia al avance temporal de un tema, a la medida de una época, o a la ponderación de un movimiento. En suma, "post" parece indicar un avance temporal que, por un lado, toma como punto de partida algo vivido y conocido anteriormente; y, por otro lado, plantea la búsqueda de lo nuevo; es decir, plantea la búsqueda de algo que está después de lo vivido y lo conocido. En este sentido, el término "post" se vincula, en primer lugar, a una normatividad establecida sobre cierto tema, época o movimiento; en segundo lugar, a la distancia crítica que pone al descubierto los límites dicha normatividad establecida; y, en tercer lugar, al avizoramiento de nuevas posibilidades o al surgimiento de potencialidades inéditas y creativas. Así, el término "post" alude al tiempo, pero no a otro tiempo que esté fuera de las coordenadas del presente, sino a un "tiempo otro", esto es, un tiempo de transformación que, desde las coordenadas propias del presente, anuncie cambios cualitativos. Veamos algunos ejemplos que ilustran el uso del prefijo "post".

El posthumanismo toma como punto de partida el humanismo clásico y desde el presente critica su normatividad interna. En este sentido, critica postulados humanistas como los siguientes: el antropocentrismo o la configuración de un universo centrado en lo humano; la creencia en la singularidad e identidad metafísica de las personas; la creencia en la superioridad de la especie animal humana respecto de otras especies animales, y respecto de otros seres inteligentes, como las máquinas biotecnológicas, por ejemplo. Frente a esta normativa, el posthumanismo postula 
una perspectiva que borre las fronteras entre lo humano, lo natural, lo animal y lo artificial, con el fin de vislumbrar nuevos horizontes de significación y abrir nuevas posibilidades de realización basadas en intervenciones y modificaciones inéditas y creativas. Según Pepperell, la condición posthumana trata sobre la evolución de la vida, la genética, la cultura y la tecnología: "si la vida puede fluir más eficientemente y moldearse mejor con sistemas mecánicos, entonces lo hará” (2003, p. 171).

Ahora veamos otro ejemplo: la postverdad. El diccionario de Oxford ha valorado la "postverdad" como la palabra del año 2016. Según este diccionario académico, la postverdad "denota circunstancias en la que los hechos objetivos influyen menos en la formación de la opinión pública, que las emociones y las creencias personales" (Flood, 2016). De acuerdo con la normativa clásica, la verdad supone una evaluación de los hechos basada en criterios objetivos. Vista de esta forma, la verdad sirve para corregir prejuicios, creencias subjetivas o posiciones emocionales. En contraste, la posverdad tiene lugar cuando la opinión pública, como la que se forma en las redes sociales, se basa no en los hechos objetivos, sino en las filiaciones emocionales y los valores grupales. En consecuencia, la novedad de la postverdad indica un cambio de época: el avizoramiento de un tiempo en el que no existen hechos objetivos, sino interpretaciones más o menos exitosas. El Brexit en Inglaterra, la elección de Trump en Estados Unidos y el plebiscito sobre la paz en Colombia son presentados como casos que vislumbran el nuevo tiempo de la posverdad (Keyes, 2004; Ramos, 2018).

Ahora dirijamos la atención al prefijo "trans". Este término parece aludir a un cambio de posición, a un dislocamiento de posiciones fijas, a un tránsito a través de algo. Comúnmente ha sido utilizado como acompañante de tópicos como los siguientes: transdisciplina, transnacional, transcultural, transgénero, transexual, entre otros. El significado del término "trans" suele estar emparentado con el significado del término "post", aunque cabe hacer una diferencia. Parece que el término "post" enfatiza más el cambio del tiempo, mientras el término "trans" enfatiza más el cambio del espacio, el cambio del lugar, el cambio de la praxis. En este sentido, el término "trans" parece referir nuevas actuaciones, las cuales expresan resistencias a normatividades pasadas o naturalizadas. Es decir, no alude a la búsqueda de algo nuevo que esté "después" de lo conocido, sino a algo concreto que encarna nuevas significaciones presentes. De ahí que el término "trans" no sólo indique el avizoramiento de lo nuevo, sino también su constatación real. De esta manera el término "trans" señala, en primer lugar, los límites de las normatividades que condicionaban la praxis pasada. En segundo lugar, el contraste significativo entre la praxis pasada y la praxis nueva; y, en tercer lugar, la puesta en obra de potencialidades inéditas y creativas. De ahí que aluda no a otro lugar que esté fuera de las coordenadas del aquí y el ahora, o a una utopía irrealizable, sino a un "lugar otro": un lugar atravesado por dinámicas cualitativas nuevas. Veamos algunos ejemplos sobre el uso del prefijo "trans".

La transdiciplina hace referencia a un nuevo modo de investigar que contrasta con el modo disciplinar e interdisciplinar. La transdiciplina concierne a lo que simultáneamente es entre las disciplinas, a través de las disciplinas y más allá de 
toda disciplina (Nicolescu, 2002). Si el objetivo de la investigación es la unidad de la realidad y la unidad del conocimiento que intenta dar cuenta de dicha realidad, entonces la normativa disciplinar tradicional resulta sesgada e insuficiente, pues hace referencia no a la realidad global sino a un aspecto particular de la realidad. Según Nicolescu (2002), la transdisciplinariedad plantea una normativa nueva basada en tres postulados, a saber: el ontológico, el lógico y el epistemológico. El postulado ontológico afirma que existen diferentes niveles de la realidad; unos niveles aluden a la realidad de los objetos, y otros niveles aluden a la realidad de los sujetos. El postulado lógico afirma que el paso de unos niveles a otros requiere el desarrollo de la lógica del tercero incluido. El postulado epistemológico afirma que la totalidad de los niveles es una estructura compleja que exige su conocimiento simultáneo. A partir de la praxis de esta normatividad, la transdisciplina constata la realización de investigaciones que armonizan perspectivas que se consideraban irreconciliables. Así, introduce nuevas actitudes y nuevas significaciones sobre antiguas antítesis, tales como: sujeto/objeto, mente/cuerpo, interno/externo, racional/causal, comprender/explicar, experiencia/experimento, emoción/razón, valor/hecho, cualidad/cantidad, verdad subjetiva/verdad objetiva, entre otras. De esta manera, la transdisciplinariedad procura una racionalidad inédita y creativa que abre las disciplinas a problemas que las atraviesan y trascienden.

Ahora veamos otro ejemplo: el transgénero. El transgénero hace referencia a un nuevo modo de comprender el género. Contrasta con la normatividad rígida según la cual el género debe ser comprendido bajo las categorías fijas "masculino" o "femenino". Este término está emparentado con el de transexualidad que comprende el sexo bajo las categorías fijas de hombre y mujer. La novedad del transgénero y del transexual radica, por un lado, en la crítica a las significaciones tradicionales naturalizadas, ya sean culturales, religiosas, científicas, filosóficas o de otra índole. Por otro lado, su novedad radica en la apertura y la realización de nuevas prácticas del género y del sexo. En este sentido, el transgénero explora la constitución de nuevas subjetividades y de nuevas significaciones que contrastan con las normatividades aportadas por las perspectivas naturalistas o metafísicas (Butler, 2006; 2007). De ahí que el género y el sexo no deban ser vistos ni como hechos naturales, ni como esencias metafísicas que predeterminan a las personas, sino constructos sociales abiertos a la realización de subjetividades otras, esto es, subjetividades capaces de autogeneración.

En síntesis, los prefijos "post" y "trans" aluden a los límites de una significación normativa establecida; a la actitud crítica, disruptiva o transgresora de dicha significación; y al vislumbramiento o constatación de nuevas significaciones, nuevas posibilidades o nuevas potencialidades. Ahora bien, los prefijos "post" y "trans parecen diferenciarse al menos en un aspecto. El prefijo "post" enfatiza una reconfiguración del tiempo, y el prefijo "trans" enfatiza una reconfiguración del espacio, del lugar o de la praxis. Ambos términos hacen referencia a movimientos que confrontan lo nuevo con lo tradicional, con lo dado, con lo antiguo, con lo habitual, con lo acostumbrado. De ahí que la promoción de los movimientos "post" y "trans" sea adjudicada a corrientes intelectuales vanguardistas que -después de la 
crítica a los grandes relatos- procuran profundas transformaciones legales, éticas, políticas, científicas, económicas; en suma, profundas transformaciones culturales.

Con base en el contexto planteado anteriormente -y sin entrar a profundizar el debate que supone el tratamiento de estos temas álgidos- proponemos argumentar la siguiente tesis: los prefijos "post" y "trans" no sólo hacen referencia a un nuevo tiempo ni a un nuevo lugar, sino también a la condición ontológica de la vida humana. Desde sus inicios hasta nuestros días la cultura ha sido "post" y "trans". Para ello, intentaremos mostrar que la vida humana es errática, lo cual no quiere decir que es errónea, sino errante (Jaramillo et al., 2018; Sáez, 2009, 2015; Villamil, 2017). En este sentido, los movimientos "post" y "trans" pueden ser comprendidos como expresiones actuales de la condición errática de la cultura. Para el desarrollo de esta tesis, planteamos el siguiente recorrido: inicialmente, presentaremos la vida humana como el dinamismo entre dos potencias heterogéneas y correlativas: la potencia céntrica y la potencia excéntrica. Luego, señalaremos los centrismos y los excentricismos culturales como patologías que ponen en crisis la cultura. Después, mostraremos el dinamismo errático de la vida humana como un criterio válido para la valoración de las culturas saludables, esto es, culturas que dinamicen tanto el poder normativo de la centricidad, como el poder creativo de la excentricidad. Finalmente, sostendremos que el cuidado y promoción del dinamismo errático de la cultura puede ser considerado como un criterio idóneo para la valoración ética de los movimientos "post" y "trans" contemporáneos.

\section{La vida humana como el dinamismo entre potencias céntricas y excéntricas}

La vida humana acontece como un juego entre fuerzas distintas, esto es, como un modo de ser discorde y dinámico que pone en obra dos potencias heterogéneas, irreductibles e inseparables, a saber: la centricidad y la excentricidad. Según Sáez (2009), "Si el hombre es un ser que tiene mundo, a diferencia de una roca, es porque lo habita céntricamente, por un lado, pero también porque es capaz de situarse excéntricamente ante lo que lo rodea, por otro lado" (p. 11). Es decir, tener mundo no alude sólo a la captación de la normatividad céntrica, sino también a la captación de sendas excéntricas que permitan recrear dicho mundo:

Si tenemos mundo, no es porque exclusivamente somos en él. Es, al mismo
tiempo, porque podemos distanciarnos excéntricamente de lo inmediato.
Para que exista una situación y sea vivida en cuanto abierta, es necesario que
podamos experimentarla con extrañeza (...) Extrañarse respecto a un lugar
mundano que nos ha hospedado hasta ese momento implica, al unísono,
colocarnos en otro lugar" (p. 54).

La potencia céntrica lleva a las personas a habitar o pertenecer a una situación significativa dada, cuyas coordenadas normativas exceden su propia capacidad de agencia. Es decir, habitar céntricamente una situación significa estar arrojado a un mundo cuyo sentido se encuentra ya constituido. La potencia excéntrica, por su parte, lleva a la persona a posicionarse fuera de la situación, a extrañarse del mundo, a ver lo que lo rodea como un campo problemático que 
requiere respuestas novedosas. La experiencia humana se conjuga en el ir y venir de estas dos potencias heterogéneas. Su movimiento errático consiste precisamente en habitar céntricamente una situación y, al mismo tiempo, consiste en trascender la experiencia excéntricamente. La potencia céntrica involucra a las personas en situaciones problemáticas, y la potencia excéntrica promueve la resolución de tales situaciones problemáticas, ya sea con la significación y normatividad establecida, o ya sea con la realización de un mundo otro.

Una analogía literaria nos puede servir de soporte para ilustrar lo dicho. Las potencias céntricas y excéntricas de la experiencia humana se parecen a las aventuras de Don Quijote y Sancho Panza. Sancho Panza representa la potencia céntrica, esto es, la inserción en una comunidad de significado con normas realistas y objetivas. Don Quijote representa la potencia excéntrica, esto es, la actitud aventurera de salir de la realidad para recrearla con nuevos significados. Ahora bien, la analogía sirve si comprendemos a Don Quijote y a Sancho Panza no como dos personas distintas, sino como dos potencias de una misma vida. Cada persona tiene la capacidad de ser céntrico como Sancho Panza y, a la vez, excéntrico como Don Quijote. Por otra parte, la analogía sirve si comprendemos a Sancho Panza no como un animal domesticado por la normatividad imperante, ni a Don Quijote como un viejo loco o esquizofrénico. Cada persona entraña hábitos adquiridos y, a la vez, la capacidad de recrear esos hábitos mediante hazañas inéditas. Pensemos en los hábitos y hazañas que supone el enamoramiento, por ejemplo.

En fin, el dinamismo errático de la vida humana emerge del conflicto y armonía de las potencias céntrica y excéntrica. De ahí que la experiencia humana pueda ser comprendida como una encrucijada. Según Sáez (2009),

\begin{abstract}
Las exigencias de la centricidad y las de la excentricidad no son opuestas, sino discordes. No es que una niegue donde la otra asiente. No es que una renuncie donde la otra emprende. No hay entre ellas una discordia reductible a la contradicción lógica (o dialéctica). Tampoco es comprensible en los términos de un conflicto entre enemigos. Más bien (...) parecen vinculadas como anverso y reverso de un único comportamiento global, de un proceso con dos vertientes heterogéneas que colisionan y trabajan extrañamente la una por la otra y a un tiempo (p. 22).
\end{abstract}

En síntesis, las potencias céntrica y excéntrica muestran la existencia humana como un ser errático, esto es, un ser en tránsito: un ser que habita normatividades, pero que puede trascenderlas de manera creativa. Ahora bien, esto no indica que la existencia humana esté a la deriva o desorientada; más bien, indica que, a cada paso, en cada época, la existencia humana es hechura y, simultáneamente, hacedora de significados.

En una línea similar a la que venimos planteando, Leonardo Boff (2002) concibe el ser humano bajo los conceptos de inmanencia y trascendencia. En nuestro lenguaje, la inmanencia hace referencia a la centricidad, y la trascendencia hace referencia a la excentricidad. Respecto de la inmanencia, Boff (2002) plantea lo siguiente: 
Somos seres enraizados y la raíz nos limita: nacemos en una familia, en una cultura, con un capital de inteligencia y de afectividad, con limitaciones físicas y sicológicas, con una dimensión patológica (porque además de ser homosapiens, también somos homo-demens). Esa es nuestra situación. Es nuestro arraigo existencial. Estamos aquí, y eso nos impide simultáneamente estar en otra parte. Estamos enraizados aquí. Esa es la dimensión de inmanencia.

De acuerdo con Boff (2002), somos seres enraizados, pero tenemos la posibilidad de trascender nuestras raíces. La trascendencia impulsa a romper barreras, a superar normas y leyes, a ir más allá de todos los límites:

Trascendencia es tal vez el desafío más secreto, más escondido del ser humano. Porque nosotros, seres humanos, hombres y mujeres, en verdad somos trascendentes. Y ser trascendentes significa ser protestantes. Protestamos continuamente. Nosotros no aceptamos la realidad en la cual estamos situados, porque somos más que todo eso, nos sentimos más. Nosotros desbordamos todos los esquemas, nada nos encaja. No hay sistema militar más duro, no hay nazismo más feroz, no hay represión eclesiástica más dogmática, no hay ciencia más exacta que pueda encuadrar al ser humano. Siempre sobra alguna cosa. No hay sistema social, por más cerrado que sea, que no tenga brechas por donde el ser humano entre y haga romper esa realidad. Por más aprisionado que esté, el ser humano siempre trasciende.

Para ilustrar la dimensión de trascendencia, Boff (2002) pone el fútbol como un ejemplo cotidiano:

La cultura popular masacrada, condenada a vivir del salario mínimo, despojada del horizonte utópico, obligada a pensar que nada va a cambiar, también tiene experiencias de trascendencia. Cuando el pobre ve que llega el día en que su equipo de fútbol va a jugar, ahí nadie lo aguanta. Hay gente que duerme para que el tiempo pase más ligero y ver a su equipo jugar. $\mathrm{Y}$ cuando su equipo hace un gol, esta persona da un salto a la trascendencia, viene el delirio, el canto, el abrazo. Es tanta la alegría que si tiene un arma y no tiene voladores ni pólvora, él hace tiros al aire. Esta es una experiencia popular de salida de sí mismo, una experiencia de trascendencia.

Con base en lo planteado por Sáez y Boff, podemos afirmar que el "post" y el "trans" hacen referencia a la potencia excéntrica o trascendente del dinamismo humano. En los ejemplos que reseñamos sobre el posthumanismo, la postverdad, la transdisciplina, el transgénero y el transexual se advierte, por un lado, la potencia céntrica, la cual alude al significado habitual y a la normatividad establecida sobre el hombre, la verdad, la disciplina, el género y el sexo. Ahí, La potencia céntrica o inmanente constituye el capital con el que contamos, el arraigo existencial que nos configura, la raíz que condiciona nuestra forma de ser en el mundo. Por otro lado, estos ejemplos indican también la potencia excéntrica o trascendente, la cual, sobre la base de la potencia céntrica o inmanente, orienta la constitución de nuevos significados; significados que protestan contra los límites rígidos de la normatividad establecida y abren nuevos modos de significación sobre el hombre, la verdad, la disciplina, el género y el sexo. Ahora bien, más allá de las diferencias conceptuales, lo importante es que para que haya dinamismo existencial se necesita el conflicto y 
la armonía entre las dos potencias. Si alguna de ellas falta, entonces el dinamismo tiende a enfermar o desaparecer.

Una analogía biológica nos ayuda a ilustrar lo dicho. Los movimientos del corazón céntricos y excéntricos, sistólicos y diastólicos, son posibles sobre la base del conflicto y la armonía entre las dos potencias. Si alguna de ellas falla, entonces el ritmo cardiaco tiende a enfermar o a morir. Este suele ser el caso de los centrismos o excentricismos que ponen en riesgo el dinamismo de la vida humana. En la medida en que estos movimientos se autonomizan o se desligan tienden a generar patologías que comprometen la dinamicidad de la vida. Veamos esto con más detalle.

\section{Los centrismos y los excentricismos culturales como patologías que ponen en crisis la vida humana.}

Como planteamos anteriormente, la existencia humana es un ser en tránsito. Esto indica que no posee ni un alfa ni un omega, ni un principio ni un fin predeterminados. Los movimientos céntricos y excéntricos hacen que la existencia humana habite y recree significaciones y normatividades. En eso consiste su vitalidad. Pero cuando se absolutiza una potencia, ya sea céntrica o excéntrica, entonces el tránsito queda obstruido o comprometido. Esta dinámica da lugar a los centrismos y a los excentricismos. Los centrismos consideran que la potencia normativa no es "una" potencia, sino "la" potencia que condiciona y determina la existencia humana. Los excéntricismos, por su parte, consideran que la potencia creativa no es "una" potencia, sino "la" potencia que dinamiza el porvenir de la existencia humana.

El problema es que sin centricidad y sin excentricidad desfallece la erótica existencial que tiende a crear vínculos y a renovarlos. El debilitamiento de los nexos entre centricidad y excentricidad constituye una verdadera disolución de la existencia humana viva. Una normatividad que no es valorada desde la experiencia humana es vacía. Una creatividad que no hace nacer una significación enriquecedora es frustrante. Sin centricidad y excentricidad nos experimentamos vacíos y frustrados. Sin centricidad y excentricidad nos experimentamos condenados a la soledad. No a esa soledad productiva que es necesaria para todo tipo de creación, sino a la soledad desoladora de quien tiene que gestionar su propia miseria sin la ayuda de nadie. Sin el dinamismo entre estas dos potencias experimentamos una pseudoexistencia en la que la estresante organización del vacío y la frustración esconde la verdad de una distensión o la disfunción fatal de potenciales. El problema es que una potencia que no interactúa con la otra no sólo se autonomiza, sino que se convierte en el agente patógeno de una enfermedad autófaga, esto es, un modo de existencia humana en el que ella se vuelve contra sí misma (Sáez, 2015). Veamos algunos ejemplos.

En relación con el centrismo, podemos considerar cómo en el mundo de la vida cotidiana se ha naturalizado una racionalización cientificista que tiende a mostrar la existencia humana como vacía y estacionaria. Esta racionalización cientificista procura una centricidad autónoma basada en un sistema de normas y 
presuntas verdades inmutables, que, desde su propia dinámica, tiende a domesticar el sentido excéntrico de una vida mejor. Ahora bien, los fenómenos de vacío y quietud no deben ser comprendidos de manera cuantitativa, sino cualitativa. Paradójicamente, en la era del vacío y en la sociedad estacionaria es cuando nos encontramos más llenos de información y más afanados de tiempo. Si la existencia humana se escinde de la potencia excéntrica, entonces comienza a organizar el vacío desde la potencia céntrica.

Pensemos, por ejemplo, en la educación o en la investigación, y en su racionalización cientificista a través de procesos de calidad y cienciometría. Sin desconocer los avances auténticos que estas actividades traen consigo, consideramos que ellas también reproducen una organización del vacío que está atada a procesos y procedimientos como los siguientes: la cosificación y cuantificación de la calidad; la formalización e instrumentalización de las experiencias de enseñanza y aprendizaje; la burocratización y administración de los procesos y procedimientos pedagógicos y didácticos; la exaltación de la dinámica competitiva del éxito; el régimen de control y vigilancia sobre los productos y los resultados educativos. Esta organización del vacío suele estar vinculada a la dinámica economicista de costo/ beneficio que opera de forma calculadora y despersonalizada. En este contexto, la novedad, la creatividad, las nuevas significaciones, en suma, la excentricidad, tiende a ser domesticada por la dinámica de la potencia céntrica. El problema es que una normatividad que no es valorada desde la experiencia humana es vacía. De ahí que este centrismo venga vinculado al malestar y el desencantamiento del mundo educativo. En esta medida, el centrismo se constituye en un agente patógeno que hace que la existencia humana se vuelva contra sí misma. No es gratuito que la Organización Mundial de la Salud considere que las enfermedades mentales son la pandemia de nuestro siglo (Alonso-Fernández, 2011); ni es gratuito que muchos de nuestros educadores sufran actualmente de depresión y de enfermedades mentales que implican desencanto y aburrimiento frente a la vida. Parafraseando a Weber, parece que nuestra cultura actual produce especialistas sin espíritu y gozadores sin corazón (Weber, 1986). Pensemos, por ejemplo, en ese tipo de educación centrista que considera que la única manera de aprender es a través del hábito, la repetición o el patrón convergente. Este tipo de educación olvida que frente al aprendizaje convergente o céntrico, se puede cultivar el aprendizaje divergente o excéntrico, el cual constituye no sólo una posibilidad sino también una oportunidad para preservar la salud de la educación.

Ahora veamos un ejemplo de excentricismo. A este tipo de experiencias, Boff (2002) las denomina experiencias de psudotrascendencia. Según Boff, la publicidad, el marketing, el negocio, el entretenimiento, la religión, la política pueden ser caldo de cultivo de experiencias de psudotrascendencia. La mayor de todas las experiencias de pseudo-trascendencia es la droga. La droga permite un viaje fantástico. La persona drogada rompe las normatividades y los límites, se siente omnipotente y vuela. El problema de la droga no es el viaje, sino el regreso del viaje. La persona ya no aguanta más la cotidianidad, el día a día, que también es inmanencia, gris, rutina, que tiene trabajo, compromisos, normas, responsabilidades, horarios. Estas 
experiencias explotan la potencia excéntrica de la existencia humana, pero no le dan la experiencia de una plenitud duradera:

La droga hace la experiencia del viaje a través de la química. Otra cosa es hacer el viaje a través del trabajo, de la búsqueda de la identidad, de un camino espiritual más largo, donde la persona conquista paso a paso los demonios que encierra en sí misma; no los endiosa ni los niega, sino que los domestica y canaliza la energía poderosa de ellos para su crecimiento, para tener una experiencia más plena de la realidad. Este proceso permite que la luz ilumine la oscuridad, que la parte sana cure la enferma. Esta experiencia de trascendencia siempre será fecunda (Boff, 2002).

Según Boff (2002), la clave para captar una experiencia de pseudotrascendencia consiste en ver que ésta siempre quiere manipular nuestra estructura de deseo. Ella canaliza toda la energía del deseo y la ata a una cosa. Luego nos muestra esa cosa como si fuera la totalidad de la realidad.

La propaganda de Marlboro nos dice que quien fuma este cigarrillo hace parte de un mundo en donde se encuentran las mujeres más espléndidas, los paisajes más espectaculares. Sólo con fumar un Marlboro, la persona accede a esa realidad. Pura mentira. Días tras día, las muchachas bonitas quieren saber menos de fumar; los paisajes bonitos cada vez más restringen el acceso a los fumadores; en consecuencia, fumar Marlboro no garantiza nada. Lo grave de este tipo de publicidad manipuladora, es que el deseo queda anclado a una ilusión, a una persona o a un objeto. Así, el deseo de la persona comienza a ser identificado con los objetos seleccionados por los publicistas.

Las experiencias excentricistas o psudotrascendentes tienden a desvincularse de la potencia céntrica. El problema es que una creatividad que no hace nacer una significación enriquecedora es frustrante. De ahí que el excentricismo esté vinculado con dos actitudes: el desencanto por la normatividad céntrica e inmanente y la frustración que producen las realidades ilusorias. El desencanto y la frustración suelen operar como agentes patógenos de la existencia humana. El auge contemporáneo de modos de ser "post" y "trans" que escapan de la realidad y vuelven empobrecidos ilustra el impacto de ciertas patologías excentricistas.

Ahora bien, los centricismos y los excentricismos nos llevan a formular una pregunta: ¿Cuál es el criterio que posibilita distinguir un dinamismo errático saludable de uno patológico? La respuesta a este interrogante supone abrirnos a actitudes dialógicas y críticas, pues no todo lo viejo es malo por ser viejo, ni todo lo nuevo es bueno por ser nuevo. ¿Qué de lo viejo y de lo nuevo nos hace mejores seres humanos? Estos interrogantes nos llevan a plantear el tercer y último momento del camino trazado.

\section{El dinamismo errático como criterio para valorar las culturas saludables}

Como hemos tratado de mostrar, la salud de la condición humana y de la cultura en general está atada a dos potencias dinámicas: la potencia normativa de la centricidad y la potencia creativa de la excentricidad. La potencia céntrica y la potencia excéntrica son conflictivas y armonizables, y en su conflictividad y 
armonización reside la salud entera de la cultura humana. Los seres humanos no estamos aquí simplemente para adaptarnos al medio ambiente o para sobrevivir, o para seguir el libreto de una normatividad; aunque la adaptación, la sobrevivencia y el cumplimiento de normas sean muy importantes. También experimentamos la llamada ética a una vida mejor, a una vida buena, a una vida excelente, a una vida abundante. Mantener viva esta diferencia de potencial es fortalecer el ritmo del corazón existencial, pues esta diferencia de potencial es el presagio de una nueva tierra para la cultura. Por consiguiente, el criterio para saber si la normatividad céntrica o la creatividad excéntrica son saludables es dinámico. El dinamismo existencial y cultural compromete el cuidado y la promoción de las dos potencias. La normatividad céntrica promueve la creatividad, y la creatividad renueva la normatividad. El florecimiento de la vida humana y de la cultura requiere, al mismo tiempo, de la conflictividad y la armonía entre la normatividad céntrica y la creatividad excéntrica. De ahí que consideremos que este criterio dinámico es errático: toma como punto de partida la normatividad de la cultura y procura creativamente una cultura mejor (Villamil, 2017).

Ahora bien, este criterio también pude ser formulado en términos negativos. Si alguna de las dos potencias desfallece, entonces ya no hay dinamismo, o el dinamismo enferma: se vuelve patológico. Si la normatividad céntrica no cuida y no promueve la creatividad excéntrica, entonces ahí es cuando comienzan los centrismos patológicos que hacen que la vida se vuelva sobre sí misma. Un centrismo patológico es aquel que toma una normatividad como la única posible, y hace de la vida una rutina estacionaria, impotente, debilitada, depotenciada. Como vimos al inicio, los movimientos "post" y "trans" suelen reaccionar frente a este tipo de centrismos patológicos denunciando la normatividad anquilosada sobre lo humano, la verdad, el género, el sexo, la disciplina, etc.

Por otro lado, si la creatividad excéntrica no cuida y no promueve la normatividad excéntrica, entonces ahí comienzan los excentricismos patológicos que hacen que la vida se vuelva contra ella misma. Un excentricismo patológico es aquel que rompe con la normatividad vigente y no procura una normatividad mejor, esto es, más acorde con las exigencias de dignidad de la vida humana. Algunos de los movimientos "post" y "trans" pueden incurrir en este craso error. Paradójicamente, una vida humana sin una normatividad de base tiende a ser estacionaria, impotente, debilitada, depotenciada. Según Boff (2002),

El criterio para saber si la trascendencia es auténtica, consiste en ver si
potencia al ser humano o lo disminuye. Hay que preguntar si la experiencia
ayuda a asumir y enriquecer la cotidianidad, o, por el contrario, representa una
fuga; si la experiencia conduce a la persona hacia la realización de su ser, o si
le da una sensación de empobrecimiento, frustración y desconcierto $(. .$.$) Las$
experiencias de pseudotrascendencia explotan la dimensión de trascendencia
del ser humano, pero no le dan la experiencia de una plenitud duradera.

En conclusión, el criterio que permite ponderar la salud o enfermedad de una existencia o de una cultura está ligado con el dinamismo errático de la potencia céntrica y la potencia excéntrica. Una vida mejor, digna y saludable requiere el cultivo 
de estas dos potencias. Una vida peor, estática y patológica implica el debilitamiento o depotenciación de la normatividad o la creatividad. El cuidado y la promoción de la normatividad céntrica y de la creatividad excéntrica constituyen la base una cultura de la vida buena en un mundo de la vida mejor. De ahí que sea urgente que los movimientos "post" y "trans" recreen las normatividades obsoletas que están a la base de prácticas contrarias al florecimiento de la vida. Qué bueno sería que el día de mañana podamos hablar de aventuras quijotescas relacionadas con la posbarbarie, la transpobreza, la posviolencia, el transegoísmo, la posarrogancia, y demás aventuras que cuiden la vulnerabilidad de la vida y promuevan su dignidad en un mundo otro.

\section{Referencias}

Alonso-Fernández, F. (2011), Factores determinantes en el trastorno depresivo en los últimos sesenta años. En: Sáez, L et al. (eds.). Occidente enfermo. Filosofía y patologias de civilización. München: Grin Verlag.

Boff, Leonardo. (2002), Tiempo de trassendencia. El ser humano como un proyecto infinito. Cantabria: Sal Terrae.

Butler, J. (2006), Deshacer el Género. Barcelona: Paidós.

Butler, J. (2007), El Género en Disputa. El Feminismo y la Subversión de la identidad. Barcelona: Paidós.

Flood, A. (2016) The Guardian, 15/11/2016. Recuperado de: https://www.theguardian. com/books/2016/nov/15/post-truth-named-word-of-the-year-by-oxford-dictionaries.

Jaramillo, C.; Cabarcas, J.; Villamil, M.; Vallejo, R.; Soto, W. (2018), "El avatar: un modo de ser cibernético cualitativamente estacionario". En: Folios, 48, pp. 193-206. DOI: http:// dx.doi.org/10.17227/folios.48-8143

Keyes, R. (2004). The Post-Truth Era. Dishonesty and Deception in Contemporary Life. Nueva York: St. Martin's Press

Nicolescu, B. (2002), Manifesto of Transdisciplinarity. New York: New York University Press.

Pepperell, R. (2003), “The Posthuman Manifesto”. En línea. Recuperado de: http://www. google.com.co/url? sa $=\mathrm{t} \& \mathrm{rct}=\mathrm{j} \& \mathrm{q}=\&$ esrc $=\mathrm{s} \&$ source $=$ web\&cd $=2 \& \mathrm{ved}=0 \mathrm{ahUKEwj-}$ j2OHuy4XWAhXCSyYKHUAbCKsQFgguMAE\&url=http $\% 3 \mathrm{~A} \% 2 \mathrm{~F} \% 2 \mathrm{Fwww}$.intellectbooks.co.uk $\% 2$ FFile $\% 3$ Adownload $\% 2$ Cid $\% 3$ D $412 \% 2$ FPepperell2.PDF\&usg=AFQjCNHP-Ei6VHmTwZedu3HpGQV0xn4WlA

Ramos, A. (2018). "La información líquida en la era de la posverdad". En: Revista General de Información y documentación, 28 (1), 283-298. DOI: http://dx.doi. org/10.5209/RGID.60809

Sáez, L. (2001), Movimientos actuales de filosofía. Madrid: Trotta. 
------- (2002), El conflicto entre analíticos y continentales. Dos tradiciones filosóficas. Barcelona: Crítica.

-------- (2009), Ser errático. Una ontología crítica de la sociedad. Madrid: Trotta.

------- (2012), “Bioexistencia. Ontología del vacío en Occidente enfermo”. En: Publicações da Escola da Agu. Direito Constitucional e Biopolitica, núm. 117, pp. 7-24.

(2015), El ocaso de Occidente. Barcelona: Herder.

------ (ed.). (2011), Occidente enfermo. Filosofia y patologías de civilización. München: Grin Verlag.

Villamil, M. (2017), Emociones humanas y ética. Para una fenomenología de las experiencias personales erráticas. Bogotá: Pontificia Universidad Javeriana.

Weber, M. (1986), El politico y el cientifico. Madrid: Alianza. 